\title{
The Development of Steering Control Strategy on Engineering Vehicles Steer-by-Wire System
}

\author{
Li Manjiang $^{1, a}$, Chang Jiangxue ${ }^{1, b}$ \\ ${ }^{1}$ Jiangsu Vocational Institute of Commerce, Nanjing 211168, China \\ alky536@126.com, ${ }^{\mathrm{b}} \mathrm{cjxsnow@126.com}$
}

Keywords: Steer-by-wire System; Engineering Vehicles; Active safety; Strategy

Abstract. Steer-by-wire is the latest technology in vehicle steering system. This paper reviews the domestic and foreign development of steer-by-wire steering system. The structure ,characteristics and working principle of the steer-by- wire steering system were analyzed in detail. At last, the solutions that making steer-by-wire steering system in engineering vehicle were proposed to improve the steering characteristics and safety of the vehicle. PID-Fuzzy control was chosen as the steering system's control algorithm, and it can realize closed-loop control of the front wheel corner.

\section{Introduction}

Trends in vehicle is safety, energy saving and environmental protection. The steering system, which affects vehicle's active safety, is very important. The quality of its stability have greatly effect on the performance of the vehicle. Steering system is a specialized body for changing the traveling direction of the vehicle, its function is to provide the best conditions in the various road sense, and transmits the intention of the driver to the vehicle. Its performance directly affects vehicle stability, safety and energy-saving environmental protection. Therefore, the study of steering system has not been interrupted.

The object of this paper is engineering vehicle's steering system. Due to poor working conditions, engineering vehicles often travel on rugged land, so there are special requirements for its steering system. Engineering vehicle's steering system mainly includes hydraulic steering and hydraulic power steering. The full hydraulic steering system has the advantage of flexibility, lightweight, performance stably and convenient layout, but there was often many faults during steering, like steering heavy and failure.

In recent years, the car began to use steer-by-wire technology. Due to the cancellation of the mechanical connection between the steering wheel and front wheel, steer-by-wire system, abbreviated as SBW, completely get rid of the limitations of traditional steering system. Not only the force transfer characteristics of the car can be freely designed, but also the angle transfer characteristics of the car, which bring infinite space to the design of the vehicle steering characteristics. This is a major innovation in automotive steering systems. SBW is one of the most advanced and cutting-edge technology in terms of steering. It is the development of the future steering system, and it can also applied to engineering vehicles.

\section{The development of SBW system at home and abroad}

SBW system was first used in aircraft control systems, it has been widely used on the plane. The driver control signal are converted into an electrical signal, and it is passed to the various execution units of the aircraft to control the attitude of the aircraft.

The study of SBW system abroad has reached a certain level. Benz began to study the front wheel steering in 1990, and applied the developed steer-by-wire system on the concept car F400 Carving; Delphi has developed its own front and four-wheel SBW system; The concept car BMWZ22 also applied steer-by-wire system, the system made a maximum range of the steering wheel designed to $160^{\circ}$, which greatly reduced the workload of the driver during emergency steering; Italian Bertone's concept car "FILO", Citroen SUV "C-Crosser", and Daimler chrysler's concept car "R129" 
have also adopted SBW system; Japan's Koyo company developed SBW system too, but in order to ensure the safety of the system, it still retained the mechanical parts between the steering wheel and the wheel, the purpose is to restore to mechanical steering when steer-by-wire system failure. The research institutions abroad were also strongly involved in the study of SBW system. Such as Stanford University's hardware in the loop test and active steering control strategy, New York State University's people-vehicles closed-loop control.

However, due to the existing regulations in Europe, the cars with steer-by-wire system which has not mechanical connection are not allowed to use on public transport road, so steer-by-wire system with purely electronic control is still in the development stage and the concept stage. With the development of electronic technology, maybe a few years later, the mechanical system will be replaced by electronic signal cable, and SBW cars will become mainstream.

Domestic study of steer-by-wire have a late start. The independently developed " the 3rd Chunhui - Claymore" mini electric car by Tong Ji University is representative. The car was on display at the 2005 Shanghai International Auto Parts \& Supplies Exhibition. Its biggest highlight was the adoption of steer-by-wire technology, and it was four-wheel-drive steer-by-wire tiny concept car; Beijing Institute of Technology have done a depth inquiry for steer-by-wire system's variable ratio; Jilin University conducted the study of the variable gear ratio, torque of the steering wheel and stability of the algorithm; Wuhan University did research on variable gear ratio and fuzzy control algorithm of road sense, and made a simulation about it. But these studies are still at an early stage, they have not been applied on a real car. In spite of it, Jilin University designed a steer-by-wire test.

\section{Structure of SBW}

Steer-by-wire system changed the original steering system's transmission structure. The system operated by the driver and the wheel's execution system were completely mechanically separated, and they made up two independent subsystems. The SBW system combined this two subsystems together by its control system and sensors. Control system take the initiative to adjust the power transfer characteristics of automotive steering by analyzing and calculating the state of the vehicle, it can also design the angle transfer characteristics of the car, which brought infinite space to the design of the vehicle steering characteristics.

As is shown in Figure1, SBW system consists of three modules: the steering wheel module, front wheel steering module and the controller module.

\section{The steering wheel module}

Steering wheel module includes steering wheel, steering wheel angle sensors, torque sensors, steering wheel aligning torque motor. The main function of the steering wheel module is to pass the driver's steering intent to the master controller, and then to control the operation of the steering module by the ECU; In the same time, the ECU send control signal to steering wheel aligning torque motor, which makes it produce the aligning torque to provide the driver with the road sense information.

\section{Front wheel steering module}

The front wheel steering module is composed of the front wheel angle sensor, steering implementation motor, steering motor controller, front wheel steering components and so on. The function of the steering assembly is to accept the master controller commands, and control the front wheel's turning by the steering motor controller, which can realize the driver's steering intent.

\section{The controller module}

The main function of the ECU is to collect sensor signal, and analyze the signal by the pre-set control strategy, and then sends commands to the steering wheel aligning torque motor and steering motor to control two motors' work. ECU should ensure that there must be the ideal vehicle response in a variety of conditions. 


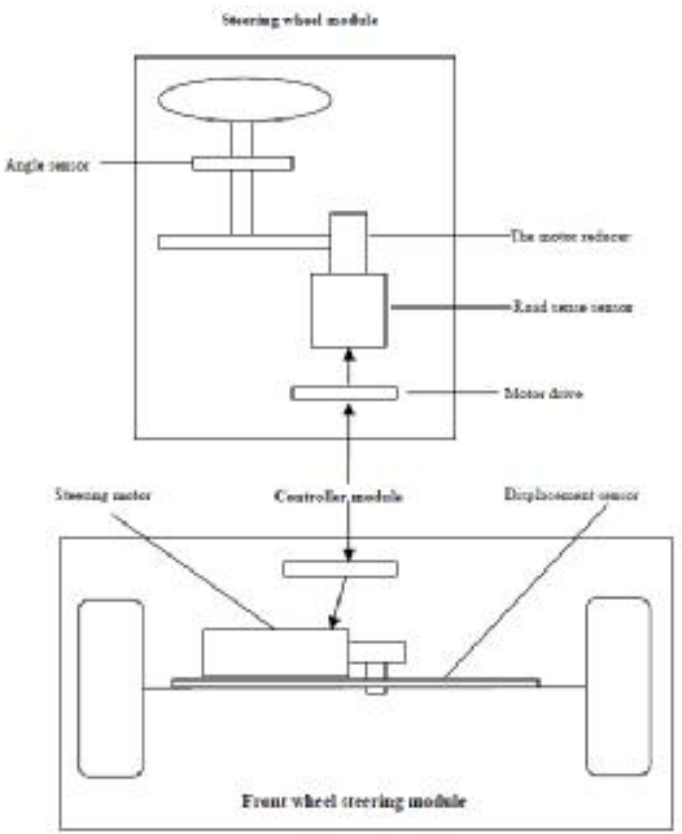

Fig.1 The structure of SBW system

\section{Tractor's Hydraulic steering by-wire system's control strategy}

Fuzzy control and PID control are two common control method in industrial control. Both methods have their advantages and disadvantages. This paper uses PID control and Fuzzy control separately to consider hydraulic steering by-wire system.

\section{The design of hydraulic steering by-wire system's PID controller}

PID controller is one of the first developed control strategies. Because of simple algorithm, high robust and reliability, it is widely used in various control systems, especially for the one which can establish a precise mathematical model. The advantages of the PID control algorithm is simple in principle, mature in technology, convenient, robust stability and wide range of applications. But there are a lot of shortcomings either, that is, the parameter requires adjustment again and again, and it is based on experience to a large extent[5].

PID controller makes the deviation of the cylinder displacement as input, and controls the electromagnetic proportional valve current by changing the proportional, integral, differential parameter, in order to achieve steering function. The control law is below:

$$
u(t)=K_{p} e(t)+K_{i} \int_{0}^{t} e(t) d t+K_{d} \frac{d e(t)}{d t}
$$

Where $\mathrm{e}(\mathrm{t})=\mathrm{r}(\mathrm{t})-\mathrm{y}(\mathrm{t}), \mathrm{K}_{\mathrm{P}}$ is a proportional parameter, $\mathrm{K}_{\mathrm{i}}$ is the integral parameter, and $\mathrm{K}_{\mathrm{d}}$ is the differential parameter.

Its control principle is shown in Fig2.

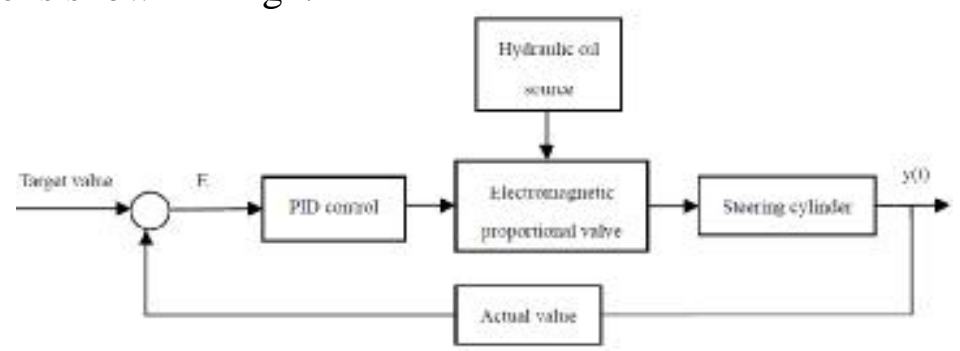

Fig. 2. The hydraulic steering by-wire system PID control principle

\section{The design of hydraulic steering by-wire system's PID controller}


With the development of technology, fuzzy control technology have become one of the most widely used and promising branches. Fuzzy controller is an expert control system, it can take advantage of existing knowledge and experience of experts and does not need to know the mathematical model of the controlled object. What's more, when the system is a non-linear system, the fuzzy controller can also produce non-linear control action. Compared with the traditional PID control, fuzzy control is particularly suitable for the system which is difficult to establish precise mathematical model, and it has characteristics of nonlinear, large time delay and complex time-varying process[6].The system makes the cylinder displacement's error and error rate of change as an input. The control principle is shown in Fig3:

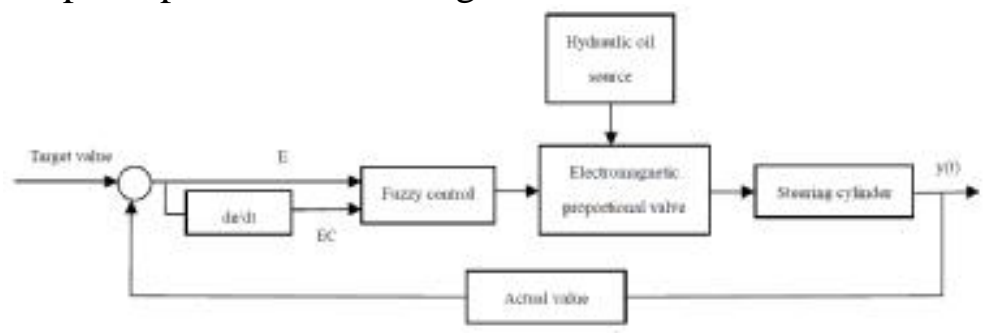

Fig. 3 The hydraulic steering by-wire system Fuzzy control principle

\section{The outlook of SBW}

With the development of technology, people have more demand for vehicle environmental protection, energy conservation, safety and comfort. However, automotive technology has been perfect, and it is difficult to change the mechanical structure of the car to make its characteristics greatly improved, so the automotive electronics technology has become the trend of modern automotive. SBW system will eventually replace the current traditional mechanical steering system, and it has gradually been accepted by the automotive industry.

As it to the engineering vehicle, if SBW is added in fully hydraulic steering system, the electrical and fluid will combine together and form a hydraulic steer-by-wire system. This will not only have the advantage of large hydraulic power, but also under the control of the electrical components, complete and flexible steering operation., which can make the steering system's structure simple, steering flexible, accurate and reliable, and improve vehicle's handling performance. It is also a subject that engineering vehicles steering system should study for.

\section{References}

[1] Hsiao-hsiang NA. Investigations on control algorithm of steady-state cornering and control strategy for dynamical correction in a steer-by-wire system. Journal of Zhejiang University(Science A:An International Applied Physics \& Engineering Journal), 2009; 10(6): 900-908.

[2] Ma Haibo, Yang Ying. Electrical power assisted steering for EVs and HEVs. Engineering Sciences, 2011; 9(1):60-65.

[3] Yu Leiyan, Lin Yi, Li Yufang.. Summary of automotive' s steer-by-wire system. Agricultural Equipment \& Vehicle Engineering, 2006.1.

[4] Zhu Yajun. Study on structure and properties of the system of electronic steering. Xi'an: Chang'an University. (2006).

[5] Li Yi-ran, Huang Jian-ming, He Zhi-ming. A Nonlinear Control Method of Steering Angle Following Used in Steer-by-wire System. Applied Mechanics and Materials. 190-191 (2012) 890-893.

[6] Liu Yong.Research of vehicles steer-by-wire system.. Wuhan University of Technology,2005.d tables must be in English. 